\title{
An Avron Rule for Fragments of R-mingle
}

\author{
George Metcalfe \\ Mathematical Institute, University of Bern \\ Sidlerstrasse 5, 3012 Bern, Switzerland \\ george.metcalfe@math.unibe.ch*
}

\begin{abstract}
Axiomatic bases of admissible rules are obtained for fragments of the substructural logic R-mingle. In particular, it is shown that a "modus-ponens-like" rule introduced by Arnon Avron forms a basis for the admissible rules of its implication and implication-fusion fragments, while a basis for the admissible rules of the full multiplicative fragment requires an additional countably infinite set of rules. Indeed, this latter case provides an example of a three-valued logic with a finitely axiomatizable consequence relation that has no finite basis for its admissible rules.
\end{abstract}

\section{Introduction}

In [5] Arnon Avron showed that the "modus-ponens-like" rule

$$
\text { (A) }\{p,(p \rightarrow(q \rightarrow q)) \rightarrow(p \rightarrow q)\} / q
$$

is admissible but not derivable in the multiplicative fragment of the substructural logic R-mingle (RM for short) (see [1-5,9,13,27]). Namely, $\{p,(p \rightarrow(q \rightarrow q)) \rightarrow(p \rightarrow q)\} \nvdash_{\mathrm{RM}} q$, but if formulas $\varphi$ and $(\varphi \rightarrow(\psi \rightarrow \psi)) \rightarrow(\varphi \rightarrow \psi)$ are theorems of this fragment, then $\psi$ is also a theorem. In this paper, it is shown that (A) in fact provides an axiomatic basis for the admissible rules of the implication and implication-fusion fragments of RM; that is, supplementing axiomatizations of these fragments with (A) provides axiomatizations of their admissible rules. However, it is also shown that to obtain a basis for the admissible rules of the full multiplicative fragment of RM, an additional countably infinite set of rules is required. Indeed, this case provides an example of a three-valued logic (axiomatized as the multiplicative fragment of RM extended with (A)) that has a finitely axiomatized consequence relation but no finite basis for its admissible rules.

In the negation-free fragment of RM, admissibility and derivability coincide; that is, this fragment is structurally complete. A proof of this result, implicit in Meyer and Slaney's proof of structural completeness for the implication-conjunction fragment of the relevant logic $\mathrm{R}$ [32], is extended here with some modifications to the implication-disjunction and implication-fusiondisjunction fragments of RM. On the other hand, the full logic RM is famously not structurally complete, being closed under the non-derivable disjunctive syllogism rule $\{p, \neg p \vee q\} / q$ (see, e.g., [1]). In other words, $\{p, \neg p \vee q\} \nvdash \mathrm{RM} q$, but whenever $\varphi$ and $\neg \varphi \vee \psi$ are theorems of RM, also $\psi$ is a theorem.

Axiomatic bases for admissible rules have been provided for a range of non-classical logics, often making crucial use of earlier foundational work by Rybakov [30,31] and Ghilardi [14, 15]. In particular, Iemhoff [16] and Rozière [29] demonstrated independently that an infinite set of "Visser rules" conjectured by De Jongh and Visser provide a basis for the admissible rules of intuitionistic logic. Bases and analytic Gentzen-style proof systems were subsequently obtained

\footnotetext{
*Supported by Swiss National Science Foundation grants 20002_129507 and 200021_146748 and Marie Curie Reintegration Grant PIRG06-GA-2009-256492.
} 
for the admissible rules of certain intermediate logics $[12,17,18]$, transitive modal logics [18, 19], and temporal logics [6,7]. For substructural logics, however, much less is known. Structural completeness for such logics and their algebras has been investigated in [11,25], but non-trivial bases have been obtained so far only for Łukasiewicz logics [20,21] and certain classes of De Morgan and Kleene algebras $[10,22]$.

\section{Preliminaries}

Let us first recall some general notions. A (propositional) language $\mathcal{L}$ is a set of connectives with specified finite arities, where the set $\mathrm{Fm}_{\mathcal{L}}$ of $\mathcal{L}$-formulas over a countably infinite set of propositional variables is defined inductively in the usual way. Arbitrary $\mathcal{L}$-formulas will be denoted by $\varphi, \psi$ and sets of $\mathcal{L}$-formulas by $\Gamma$. An $\mathcal{L}$-substitution $\sigma$ is an endomorphism on the formula algebra $\mathbf{F m}_{\mathcal{L}}$, writing $\sigma(\Gamma)$ for $\{\sigma(\varphi) \mid \varphi \in \Gamma\}$.

A logic $\mathrm{L} \subseteq \mathcal{P}\left(\mathrm{Fm}_{\mathcal{L}}\right) \times \mathrm{Fm}_{\mathcal{L}}$ in the language $\mathcal{L}$ is a finitary structural consequence relation on $\mathbf{F m}_{\mathcal{L}}$; that is, writing $\Gamma \vdash_{\mathrm{L}} \varphi$ for $(\Gamma, \varphi) \in \mathrm{L}$ :

- $\{\varphi\} \vdash_{\mathrm{L}} \varphi$.

- If $\Gamma \vdash_{\mathrm{L}} \varphi$ and $\Gamma \subseteq \Gamma^{\prime}$, then $\Gamma^{\prime} \vdash_{\mathrm{L}} \varphi$.

- If $\Gamma \vdash_{\mathrm{L}} \varphi$ and $\Gamma \cup\{\varphi\} \vdash_{\mathrm{L}} \psi$, then $\Gamma \vdash_{\mathrm{L}} \psi$.

- If $\Gamma \vdash_{\mathrm{L}} \varphi$, then $\sigma(\Gamma) \vdash_{\mathrm{L}} \sigma(\varphi)$ for each $\mathcal{L}$-substitution $\sigma$.

- If $\Gamma \vdash_{\mathrm{L}} \varphi$, then there is a finite set $\Gamma^{\prime} \subseteq \Gamma$ such that $\Gamma^{\prime} \vdash_{\mathrm{L}} \varphi$.

An L-theorem is a formula $\varphi$ such that $\emptyset \vdash_{\mathrm{L}} \varphi\left(\right.$ abbreviated to $\left.\vdash_{\mathrm{L}} \varphi\right)$.

Let $\mathrm{L}_{1}$ be a logic in a language $\mathcal{L}_{1}$. A logic $\mathrm{L}_{2}$ in a language $\mathcal{L}_{2} \supseteq \mathcal{L}_{1}$ is called an expansion of $\mathrm{L}_{1}$, and an extension if $\mathcal{L}_{1}$ and $\mathcal{L}_{2}$ coincide, if for all $\Gamma \cup\{\varphi\} \subseteq \operatorname{Fm}_{\mathcal{L}_{1}}: \Gamma \vdash_{\mathrm{L}_{1}} \varphi \operatorname{implies} \Gamma \vdash_{\mathrm{L}_{2}} \varphi$. Such an expansion is called conservative if $\Gamma \vdash_{L_{2}} \varphi$ implies $\Gamma \vdash_{L_{1}} \varphi$ for each $\Gamma \cup\{\varphi\} \subseteq \mathrm{Fm}_{\mathcal{L}_{1}}$. In this case, $\mathrm{L}_{1}$ is the (unique) $\mathcal{L}_{1}$-fragment of $\mathrm{L}_{2}$, denoted by $\mathrm{L}_{2} \mid \mathcal{L}_{1}$.

A logic L is called structurally complete if all of its extensions have new theorems, and hereditarily structurally complete if all of its extensions are structurally complete. Moreover, let us define a rule for a language $\mathcal{L}$ to be an ordered pair $\Gamma / \varphi$, where $\Gamma \cup\{\varphi\} \subseteq \operatorname{Fm}_{\mathcal{L}}$ is finite. $\Gamma / \varphi$ is called L-derivable if $\Gamma \vdash_{\mathrm{L}} \varphi$, and L-admissible if for each $\mathcal{L}$-substitution $\sigma$, whenever $\vdash_{\mathrm{L}} \sigma(\psi)$ for all $\psi \in \Gamma$, then $\vdash_{\mathrm{L}} \sigma(\varphi)$. It follows that $\mathrm{L}$ is structurally complete if and only if (henceforth, iff) every L-admissible rule is L-derivable (see, e.g., [25,31]).

Now define for $\Gamma \cup\{\varphi\} \subseteq \mathrm{Fm}_{\mathcal{L}}$ :

$$
\Gamma \sim_{\mathrm{L}} \varphi \text { iff } \Gamma^{\prime} / \varphi \text { is L-admissible for some finite } \Gamma^{\prime} \subseteq \Gamma .
$$

Then $\sim_{\mathrm{L}}=\left\{(\Gamma, \varphi) \in \mathcal{P}\left(\mathrm{Fm}_{\mathcal{L}}\right) \times \mathrm{Fm}_{\mathcal{L}} \mid \Gamma \sim_{\mathrm{L}} \varphi\right\}$ is also a logic. For a set of rules $\mathcal{R}$, let $\mathrm{L}+\mathcal{R}$ denote the smallest logic containing $\mathrm{L} \cup \mathcal{R}$, writing $\mathrm{L}+r_{1}+\ldots+r_{n}$ if $\mathcal{R}=\left\{r_{1}, \ldots, r_{n}\right\}$. The set $\mathcal{R}$ is called a basis for the admissible rules of $\mathrm{L}$ if $\mathrm{L}+\mathcal{R}$ is $\sim_{\mathrm{L}}$.

The following simple lemma will play a key role in our proofs that a given set of rules forms a basis for the admissible rules of a logic.

Lemma 2.1. Let $\mathrm{L}$ be a logic and let $\mathcal{R}$ be a set of $\mathrm{L}$-admissible rules such that $\mathrm{L}+\mathcal{R}$ is structurally complete. Then $\mathcal{R}$ is a basis for the admissible rules of $\mathrm{L}$.

Proof. Suppose that $\mathrm{L}+\mathcal{R}$ is structurally complete. Then $\Gamma \vdash_{\mathrm{L}+\mathcal{R}} \varphi$ implies $\Gamma \sim_{\mathrm{L}} \varphi$ since $\sim_{\mathrm{L}}$ is a logic containing $\mathrm{L} \cup \mathcal{R}$. Suppose now that $\Gamma \sim_{\mathrm{L}} \varphi$. If $\vdash_{\mathrm{L}+\mathcal{R}} \sigma(\psi)$ for all $\psi \in \Gamma$, then $\sim_{\mathrm{L}} \sigma(\psi)$ for all $\psi \in \Gamma$, so $\vdash_{\mathrm{L}} \sigma(\varphi)$ and therefore also $\vdash_{\mathrm{L}+\mathcal{R}} \sigma(\varphi)$. Hence $\Gamma \sim_{\mathrm{L}+\mathcal{R}} \varphi$. But $\mathrm{L}+\mathcal{R}$ is structurally complete, so also $\Gamma \vdash_{\mathrm{L}+\mathcal{R}} \varphi$. 
Let us now consider the algebraic setting. We fix $\mathrm{K}$ to be a class of algebraic structures (algebras) of the same language $\mathcal{L}$. For a set of $\mathcal{L}$-identities $\Sigma \cup\{\varphi \approx \psi\}$, we write $\Sigma \models_{\mathrm{K}} \varphi \approx \psi$ if for each $\mathbf{A} \in \mathrm{K}$ and homomorphism $h: \mathbf{F m}_{\mathcal{L}} \rightarrow \mathbf{A}$, whenever $h\left(\varphi^{\prime}\right)=h\left(\psi^{\prime}\right)$ for all $\varphi^{\prime} \in \psi^{\prime} \in \Sigma$, also $h(\varphi)=h(\psi)$. We say that $\mathrm{K}$ is a (quasi)variety if it is axiomatizable by a set of (quasi-)identities. We also define the variety $\mathbb{V}(\mathrm{K})$ and quasivariety $\mathbb{Q}(\mathrm{K})$ generated by $\mathrm{K}$ to be the smallest variety and quasivariety containing $\mathrm{K}$, respectively.

The logical and algebraic settings are connected via the theory of algebraizable logics (see [8]). A logic $\mathrm{L}$ is algebraizable if there is a quasivariety $\mathrm{K}$ together with a set of identities in one variable $E(p)$ and a set of formulas in two variables $D(p, q)$ (called translations) satisfying

1. $\Gamma \vdash_{\mathrm{L}} \varphi$ iff $\bigcup\{E(\psi) \mid \psi \in \Gamma\} \models_{\mathrm{K}} E(\varphi)$.

2. $\Sigma \models_{\mathrm{K}} \varphi \approx \psi$ iff $\bigcup\left\{D\left(\varphi^{\prime}, \psi^{\prime}\right) \mid \varphi^{\prime} \approx \psi^{\prime} \in \Sigma\right\} \vdash_{\mathrm{L}} D(\varphi, \psi)$.

3. $\bigcup\left\{D\left(\varphi^{\prime}, \psi^{\prime}\right) \mid \varphi^{\prime} \approx \psi^{\prime} \in E(\varphi)\right\} \vdash_{\mathrm{L}} \varphi$ and $\{\varphi\} \vdash_{\mathrm{L}} D\left(\varphi^{\prime}, \psi^{\prime}\right)$ for each $\varphi^{\prime} \approx \psi^{\prime} \in E(\varphi)$.

4. $\bigcup\left\{E\left(\varphi^{\prime}\right) \mid \varphi^{\prime} \in D(\varphi, \psi)\right\} \models_{\mathrm{K}} \varphi \approx \psi$ and $\{\varphi \approx \psi\} \models_{\mathrm{K}} E\left(\varphi^{\prime}\right)$ for each $\varphi^{\prime} \in D(\varphi, \psi)$.

$\mathrm{K}$ is then called an equivalent quasivariety of $\mathrm{L}$.

The following theorem is very helpful for dealing with fragments of logics. Recall that an $\mathcal{L}$-subreduct of an algebra $\mathbf{A}$ is a subalgebra of an $\mathcal{L}$-reduct of $\mathbf{A}$.

Theorem 2.2 ([8]). Let $\mathrm{L}$ be an algebraizable logic for a language $\mathcal{L}$ with an equivalent quasivariety $\mathrm{Q}$ and translations in $\mathcal{L}^{\prime} \subseteq \mathcal{L}$. Then $\mathrm{L}\left\lceil\mathcal{L}^{\prime}\right.$ is algebraizable with the same translations and has an equivalent quasivariety $\mathrm{Q} \mid \mathcal{L}^{\prime}$ consisting of all $\mathcal{L}^{\prime}$-subreducts of algebras from $\mathrm{Q}$.

Given an algebraizable logic $\mathrm{L}$ with equivalent quasivariety $\mathrm{Q}$, the algebraic notion corresponding to an L-admissible rule is a quasi-identity that holds in the free algebra of $\mathrm{Q}$ on countably infinitely many generators, denoted by $\mathbf{F}_{\mathrm{Q}}$ (see, e.g., [31]). Below, we recall a useful simple condition (a particular case of a theorem from [11]) for the logic of a quasivariety generated by a single algebra to be structurally complete. We make use of the canonical morphism $h_{\mathrm{Q}}: \mathbf{F m}_{\mathcal{L}} \rightarrow \mathbf{F}_{\mathrm{Q}}$ which maps each formula $\varphi$ to its equivalence class in the free algebra.

Lemma 2.3 ([11]). Let L be a logic for a language $\mathcal{L}$ with an equivalent quasivariety $\mathbb{Q}(\mathbf{A})$ such that $h_{\mathbb{Q}(\mathbf{A})} \circ g$ embeds $\mathbf{A}$ into $\mathbf{F}_{\mathbb{Q}(\mathbf{A})}$ for some map $g: A \rightarrow \mathrm{Fm}_{\mathcal{L}^{\prime}}$ and $\mathcal{L}^{\prime} \subseteq \mathcal{L}$. Then $\mathrm{L}\left\lceil\mathcal{L}^{\prime}\right.$ is structurally complete.

\section{R-mingle and its fragments}

The logic R-mingle (for short, RM) is traditionally formulated in a language with the binary connectives $\rightarrow$ (implication), $\wedge$ (conjunction), and $\vee$ (disjunction), and the unary connective $\neg$ (negation). However, in the landscape of substructural logics, the "multiplicative" conjunction . (fusion), definable in $\mathrm{RM}$ as $\varphi \cdot \psi==_{\text {def }} \neg(\varphi \rightarrow \neg \psi)$, plays an important role as the partner of its residuum $\rightarrow$. An axiomatization of $\mathrm{RM}$ in this extended language is provided in Figure 1 . As usual, we identify axiomatizations with their associated consequence relations.

Consider the following binary functions on the set of integers $\mathbb{Z}$, where $\wedge$ and $\vee$ are min and max, respectively, $\neg$ is the usual unary minus operation, and $|x|$ is the absolute value of $x$ :

$$
x \cdot y=\left\{\begin{array}{ll}
x \wedge y & \text { if }|x|=|y| \\
y & \text { if }|x|<|y| \\
x & \text { if }|y|<|x|
\end{array} \quad x \rightarrow y= \begin{cases}\neg x \vee y \text { if } x \leq y \\
\neg x \wedge y \text { otherwise. }\end{cases}\right.
$$

Let $\mathbf{Z}$ be the algebra $\langle\mathbb{Z}, \rightarrow, \cdot \wedge, \vee, \neg\rangle$. We denote by $\mathrm{SA}$ the quasivariety $\mathbb{Q}(\mathbf{Z})$ of Sugihara algebras, noting that this name is used in [9] for the same algebras without the (definable) fusion operation. Sugihara algebras may also be characterized as $t$-free subreducts of distributive idempotent involutive commutative residuated lattices (see, e.g., [27] for details). 

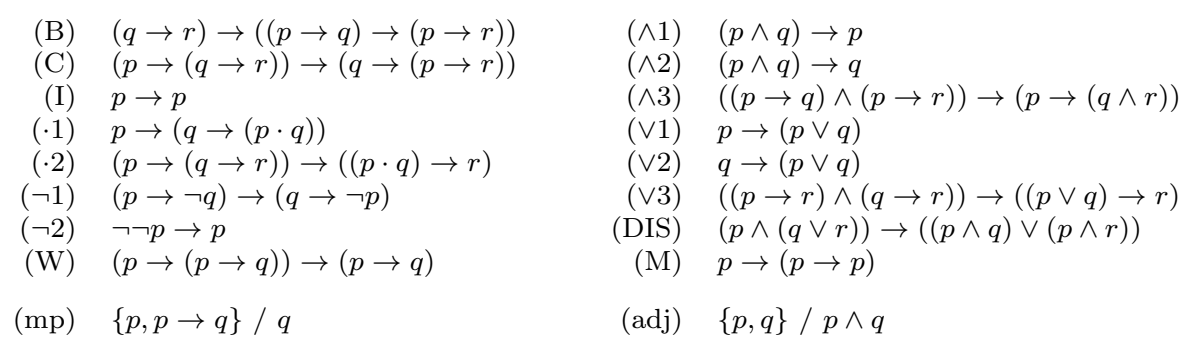

Figure 1: An axiomatization of RM.

For any Sugihara algebra $\mathbf{A}$ and $x \in A$, let us fix $|x|=_{\text {def }} x \rightarrow x$. Then the lattice order of $\mathbf{A}$ is equationally definable for $x, y \in A$ by

$$
x \leq y \quad \text { iff } \quad x \rightarrow y=|x \rightarrow y| .
$$

Note in particular that $0 \leq x$ in $\mathbf{Z}$ iff $x=|x|$.

Taking advantage of this formulation, which covers all fragments considered in this paper, let us define for $\{\rightarrow\} \subseteq \mathcal{L} \subseteq\{\rightarrow, \cdot, \wedge, \vee, \neg\}$, a class of $\mathcal{L}$-subreducts of Sugihara algebras $\mathrm{K}$, and $\Gamma \cup\{\varphi\} \subseteq \mathrm{Fm}_{\mathcal{L}}:$

$$
\Gamma \models_{\mathrm{K}} \varphi \quad \text { iff } \quad\{\psi \approx|\psi| \mid \psi \in \Gamma\} \models_{\mathrm{K}} \varphi \approx|\varphi|
$$

abbreviating $\Gamma \models_{\{\mathbf{A}\}} \varphi$ to $\Gamma \models_{\mathbf{A}} \varphi$. Then RM has the following strong completeness theorem:

Theorem 3.1 (Meyer, Dunn [1,13]). For $\{\rightarrow\} \subseteq \mathcal{L} \subseteq\{\rightarrow, \cdot \wedge, \vee, \neg\}$ and $\Gamma \cup\{\varphi\} \subseteq \operatorname{Fm}_{\mathcal{L}}$ :

$$
\Gamma \vdash_{\mathrm{RM}} \varphi \quad \text { iff } \quad \Gamma \models \mathrm{SA} \varphi \text {. }
$$

Note also that for any set of identities $\Sigma \cup\{\varphi \approx \psi\}$ for $\mathcal{L}$ :

$$
\begin{array}{ll}
\Sigma=\mathrm{SA} \varphi \approx \psi \quad \text { iff } \quad & \bigcup_{\varphi^{\prime} \approx \psi^{\prime} \in \Sigma}\left\{\varphi^{\prime} \rightarrow \psi^{\prime}, \psi^{\prime} \rightarrow \varphi^{\prime}\right\} \vdash_{\mathrm{RM}} \varphi \rightarrow \psi \\
& \text { and } \bigcup_{\varphi^{\prime} \approx \psi^{\prime} \in \Sigma}\left\{\varphi^{\prime} \rightarrow \psi^{\prime}, \psi^{\prime} \rightarrow \varphi^{\prime}\right\} \vdash_{\mathrm{RM}} \psi \rightarrow \varphi .
\end{array}
$$

That is, SA is an equivalent quasivariety for the logic $\mathrm{RM}$ with translations $E(p)=\{p \approx|p|\}$ and $D(p, q)=\{p \rightarrow q, q \rightarrow p\}$. Moreover, by Lemma 2.2, we obtain equivalent quasivarieties for all fragments of RM with implication.

To see now that RM and some of its fragments enjoy a deduction theorem, consider the following connective introduced by Avron in [2]:

$$
\varphi \supset \psi={ }_{\text {def }}(\varphi \rightarrow \psi) \vee \psi .
$$

Theorem $3.2([2]) . \Gamma \cup\{\varphi\} \vdash_{\mathrm{RM}} \psi \quad$ iff $\quad \Gamma \vdash_{\mathrm{RM}} \varphi \supset \psi$.

It is easily checked that the following identities hold in all Sugihara algebras, either by calculation in the algebra $\mathbf{Z}$ or by providing derivations in Avron's elegant hypersequent calculus for RM [3]:

$$
\begin{aligned}
x \wedge y & \approx \neg(\neg x \vee \neg y) \\
x \vee y & \approx \neg(\neg x \wedge \neg y) \\
x \cdot y & \approx \neg(x \rightarrow \neg y) \\
x \supset y & \approx(x \wedge|y|) \rightarrow y \\
x \vee y & \approx((x \rightarrow y) \supset y) \wedge((y \rightarrow x) \supset x) \\
x \cdot y & \approx((x \rightarrow|y|) \rightarrow y) \wedge((y \rightarrow|x|) \rightarrow x) .
\end{aligned}
$$

The first three identities are well-known and hold in all involutive commutative residuated lattices, while the fourth and fifth have appeared already in [9]. The last identity, which shows that fusion is definable using implication and conjunction, seems to be new, however. Together, they allow us to distinguish the various superimplicational (i.e., containing implication) fragments of RM (see Figure 2). 


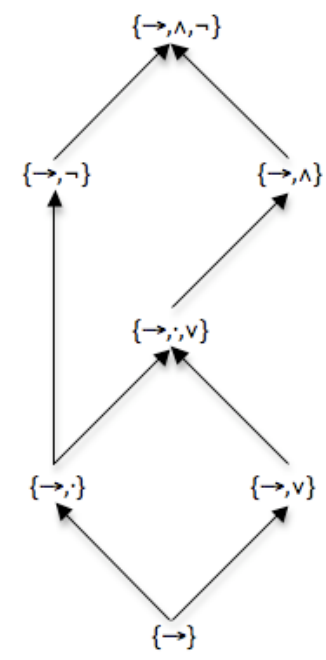

Figure 2: Superimplicational fragments of RM.

Theorem 3.3. The distinct superimplicational fragments of RM consist of the following sets of connectives:

$$
\{\rightarrow, \wedge, \neg\} \quad\{\rightarrow, \wedge\} \quad\{\rightarrow, \cdot, \vee\} \quad\{\rightarrow, \vee\} \quad\{\rightarrow, \neg\} \quad\{\rightarrow, \cdot\} \quad\{\rightarrow\} .
$$

Proof. It follows directly from the identities listed above that these sets of connectives exhaust the possibilities for the various superimplicational fragments of RM. In particular the $\{\rightarrow, \wedge, \neg\}$ fragment coincides with the whole of RM and the $\{\rightarrow, \wedge\}$-fragment is the positive fragment of RM. Hence the diagram in Figure 2 represents all inclusions between fragments of RM and it remains to check that these inclusions are proper.

Note first that it is shown in [9] that the $\{\rightarrow, \wedge, \neg\},\{\rightarrow, \wedge\},\{\rightarrow, \vee\},\{\rightarrow, \neg\}$, and $\{\rightarrow\}$ fragments are distinct. Moreover, since classical logic is an axiomatic extension of RM where $\wedge$ and - collapse, and the implication, implication-negation, and implication-conjunction fragments of classical logic are distinct, it follows also that the $\{\rightarrow\},\{\rightarrow, \cdot\},\{\rightarrow, \vee\},\{\rightarrow, \cdot, \vee\}$, and $\{\rightarrow, \neg\}-$ fragments of RM are distinct.

Finally, we show that the $\{\rightarrow, \cdot, \vee\}$ and $\{\rightarrow, \wedge\}$ fragments are distinct by considering the subalgebra of the $\{\rightarrow, \cdot, \vee V\}$-reduct of $\mathbf{Z} \times \mathbf{Z}$ generated by $(1,0)$ and $(0,1)$. This subalgebra contains the elements $(1,1),(1,0),(0,1),(1,-1),(-1,1)$, and $(-1,-1)$, but not the meet $(0,0)$ of $(1,0)$ and $(0,1)$. Hence $\wedge$ is not definable in the $\{\rightarrow, \cdot, \vee\}$-fragment.

Following [9], we call superimplicational fragments of RM with at least one of $\wedge$ and $\vee$, upper fragments, and the rest, lower fragments.

\section{The Upper Fragments}

Certain positive (lacking negation) upper fragments of RM are hereditarily structurally complete. Indeed, this was established implicitly for the $\{\rightarrow, \wedge\}$-fragment of RM in [32] (see also [25]), although to the best of our knowledge, the $\{\rightarrow, \vee\}$ and $\{\rightarrow, \cdot, \vee\}$ fragments have not been considered in the literature. Here we adapt the approach of [32] to obtain a uniform hereditary structural completeness proof for all three of these fragments.

Theorem 4.1. The $\{\rightarrow, \vee\},\{\rightarrow, \cdot, \vee\}$, and $\{\rightarrow, \wedge\}$ fragments of $\mathrm{RM}$ are hereditarily structurally complete. 
Proof. Let $\left\{\varphi_{1}, \ldots, \varphi_{n}\right\}$ be a finite set of formulas for one of these fragments. We write $\vec{\varphi} \supset \psi$ for the formula $\varphi_{1} \supset\left(\varphi_{2} \supset \ldots \supset\left(\varphi_{n} \supset \psi\right) \ldots\right)$, and define the substitution:

$$
\sigma(p)=\vec{\varphi} \supset p
$$

We prove by induction on formula complexity that for all $\psi \in \mathrm{Fm}_{\{\rightarrow, \cdot, \wedge, \vee\}}$ :

$$
\vdash_{\mathrm{RM}} \sigma(\psi) \rightarrow(\vec{\varphi} \supset \psi) \text { and } \quad \vdash_{\mathrm{RM}}(\vec{\varphi} \supset \psi) \rightarrow \sigma(\psi) \text {. }
$$

The base case is immediate. For the inductive step, assume for $i=1,2$ that $\vdash_{\mathrm{RM}} \sigma\left(\psi_{i}\right) \rightarrow\left(\vec{\varphi} \supset \psi_{i}\right)$ and $\vdash_{\mathrm{RM}}\left(\vec{\varphi} \supset \psi_{i}\right) \rightarrow \sigma\left(\psi_{i}\right)$. Then for each connective $\# \in\{\rightarrow, \cdot, \wedge, \vee\}$ :

$$
\vdash_{\mathrm{RM}} \sigma\left(\psi_{1} \# \psi_{2}\right) \rightarrow\left(\left(\vec{\varphi} \supset \psi_{1}\right) \#\left(\vec{\varphi} \supset \psi_{2}\right)\right) \quad \text { and } \quad \vdash_{\mathrm{RM}}\left(\left(\vec{\varphi} \supset \psi_{1}\right) \#\left(\vec{\varphi} \supset \psi_{2}\right)\right) \rightarrow \sigma\left(\psi_{1} \# \psi_{2}\right) .
$$

But also (using, e.g., Avron's hypersequent calculus [3])

$$
\begin{aligned}
& \vdash_{\mathrm{RM}}\left(\left(\vec{\varphi} \supset \psi_{1}\right) \rightarrow\left(\vec{\varphi} \supset \psi_{2}\right)\right) \rightarrow\left(\vec{\varphi} \supset\left(\psi_{1} \rightarrow \psi_{2}\right)\right) \\
& \vdash_{\mathrm{RM}}\left(\vec{\varphi} \supset\left(\psi_{1} \rightarrow \psi_{2}\right)\right) \rightarrow\left(\left(\vec{\varphi} \supset \psi_{1}\right) \rightarrow\left(\vec{\varphi} \supset \psi_{2}\right)\right) \\
& \vdash_{\mathrm{RM}}\left(\left(\vec{\varphi} \supset \psi_{1}\right) \cdot\left(\vec{\varphi} \supset \psi_{2}\right)\right) \rightarrow\left(\vec{\varphi} \supset\left(\psi_{1} \cdot \psi_{2}\right)\right) \\
& \vdash_{\mathrm{RM}}\left(\vec{\varphi} \supset\left(\psi_{1} \cdot \psi_{2}\right)\right) \rightarrow\left(\left(\vec{\varphi} \supset \psi_{1}\right) \cdot\left(\vec{\varphi} \supset \psi_{2}\right)\right) \\
& \vdash_{\mathrm{RM}}\left(\left(\vec{\varphi} \supset \psi_{1}\right) \wedge\left(\vec{\varphi} \supset \psi_{2}\right)\right) \rightarrow\left(\vec{\varphi} \supset\left(\psi_{1} \wedge \psi_{2}\right)\right) \\
& \vdash_{\mathrm{RM}}\left(\vec{\varphi} \supset\left(\psi_{1} \wedge \psi_{2}\right)\right) \rightarrow\left(\left(\vec{\varphi} \supset \psi_{1}\right) \wedge\left(\vec{\varphi} \supset \psi_{2}\right)\right) \\
& \vdash_{\mathrm{RM}}\left(\left(\vec{\varphi} \supset \psi_{1}\right) \vee\left(\vec{\varphi} \supset \psi_{2}\right)\right) \rightarrow\left(\vec{\varphi} \supset\left(\psi_{1} \vee \psi_{2}\right)\right) \\
& \vdash_{\mathrm{RM}}\left(\vec{\varphi} \supset\left(\psi_{1} \vee \psi_{2}\right)\right) \rightarrow\left(\left(\vec{\varphi} \supset \psi_{1}\right) \vee\left(\vec{\varphi} \supset \psi_{2}\right)\right) .
\end{aligned}
$$

Hence for each $\# \in\{\rightarrow, \cdot, \wedge, \vee\}$, as required:

$$
\vdash_{\mathrm{RM}} \sigma\left(\psi_{1} \# \psi_{2}\right) \rightarrow\left(\vec{\varphi} \supset\left(\psi_{1} \# \psi_{2}\right)\right) \quad \text { and } \quad \vdash_{\mathrm{RM}}\left(\vec{\varphi} \supset\left(\psi_{1} \# \psi_{2}\right)\right) \rightarrow \sigma\left(\psi_{1} \# \psi_{2}\right) \text {. }
$$

Now for each $i=1 \ldots n$, since $\vdash_{\mathrm{RM}} \vec{\varphi} \supset \varphi_{i}$, also, using the above claim, $\vdash_{\mathrm{RM}} \sigma\left(\varphi_{i}\right)$. Moreover, since $\vdash_{\mathrm{RM}} \sigma(\psi) \rightarrow(\vec{\varphi} \supset \psi)$, it follows that $\vdash_{\mathrm{RM}} \vec{\varphi} \supset(\sigma(\psi) \rightarrow \psi)$ and hence, by the deduction theorem for $\supset$ (Theorem 3.2), that $\left\{\varphi_{1}, \ldots, \varphi_{n}\right\} \vdash_{\mathrm{RM}} \sigma(\psi) \rightarrow \psi$. Suppose finally that $\mathrm{L}$ is an extension of the $\{\rightarrow, \vee\},\{\rightarrow, \cdot, \vee\}$, or $\{\rightarrow, \wedge\}$ fragment of RM, and that $\left\{\varphi_{1}, \ldots, \varphi_{n}\right\} / \psi$ is an L-admissible rule. Since $\vdash_{\mathrm{L}} \sigma\left(\varphi_{i}\right)$ for $i=1 \ldots n$, also $\vdash_{\mathrm{L}} \sigma(\psi)$. But $\left\{\varphi_{1}, \ldots, \varphi_{n}\right\} \vdash_{\mathrm{L}} \sigma(\psi) \rightarrow \psi$, so using modus ponens, $\left\{\varphi_{1}, \ldots, \varphi_{n}\right\} \vdash_{\mathrm{L}} \psi$ as required. That is, $\mathrm{L}$ is structurally complete. So the $\{\rightarrow, \vee\},\{\rightarrow, \cdot, \vee\}$, and $\{\rightarrow, \wedge\}$ fragments of RM are hereditarily structurally complete.

The strategy described in the above proof was first used by Prucnal in [26] to prove hereditary structural completeness for the implication fragment of each intermediate logic, and is hence often referred to as "Prucnal's trick". In fact, the proof establishes that each finite set of formulas $\Gamma$ in the considered fragment $\mathrm{L}$ is L-projective, the substitution $\sigma$ being a so-called L-projective unifier of $\Gamma$ : namely $\vdash_{\mathrm{L}} \sigma(\psi)$ for all $\psi \in \Gamma$ and for each variable $p, \Gamma \vdash_{\mathrm{L}} p \rightarrow \sigma(p)$ and $\Gamma \vdash_{\mathrm{L}} \sigma(p) \rightarrow$ $p$ (see $[14,15]$ for further details). We note also that a more complicated proof of hereditary structural completeness has been provided by Olson and Raftery in [24] for the positive fragment of $\mathrm{RM}^{\mathrm{t}}$, axiomatized by extending an axiomatization for the positive fragment of RM with axioms $p \rightarrow(t \rightarrow p)$ and $t$ for the constant $t$. 


\section{The Lower Fragments}

We turn our attention now to the lower fragments of $\mathrm{RM}$ : the implication fragment $\operatorname{RM}\lceil\{\rightarrow\}$, the implication-fusion fragment $\operatorname{RM}\lceil\{\rightarrow, \cdot\}$, and the multiplicative fragment $\operatorname{RM} \uparrow\{\rightarrow, \cdot, \neg\}$. In this setting, the following algebra (the $\{\rightarrow, \cdot, \neg\}$-reduct of a three element Sugihara algebra) plays a pivotal role:

$$
\mathbf{S}=\langle\{-1,0,1\}, \rightarrow, \cdot, \neg\rangle
$$

where $\rightarrow, \cdot$, and $\neg$ are the operations of $\mathbf{Z}$ restricted to $\{-1,0,1\}$. Recall that (as in $\mathbf{Z}$ ) $x \geq 0$ in $\mathbf{S}$ iff $x=|x|$. So in particular, $\Gamma \models_{\mathbf{S}} \varphi$ iff for every homomorphism $h: \mathbf{F m}_{\{\rightarrow, \cdot, \neg\}} \rightarrow \mathbf{S}$, whenever $h(\psi) \geq 0$ for all $\psi \in \Gamma$, also $h(\varphi) \geq 0$.

For convenience, let us denote the multiplicative fragment $\operatorname{RM}\left\lceil\{\rightarrow, \cdot, \neg\}\right.$ by $\operatorname{RM}_{m}$ and its equivalent quasivariety by $\mathrm{SA}_{\mathrm{m}}$. The following key result for this fragment was established by Sobociński in [33] (see also [3] and [9] for proof-theoretic and algebraic proofs, respectively):

Theorem $5.1([33]) \cdot \mathbb{V}\left(\mathrm{SA}_{\mathrm{m}}\right)=\mathbb{V}(\mathrm{S})$.

However, consider now Avron's defined implication

$$
\varphi \Rightarrow \psi={ }_{\text {def }}(\varphi \rightarrow|\psi|) \rightarrow(\varphi \rightarrow \psi)
$$

and the modus ponens rule for this connective

$$
\text { (A) }\{p, p \Rightarrow q\} / q \text {. }
$$

Then $\{p, p \Rightarrow q\} \quad \forall \mathrm{SA}_{\mathrm{m}} q$ : just consider the $\{\rightarrow, \cdot, \neg\}$-reduct of $\mathbf{Z}$ and evaluate $p$ as 2 and $q$ as -1 . But $\{p, p \Rightarrow q\} \models \mathbf{S} q$. So $\mathbf{S A}_{\mathbf{m}}$ is not generated by $\mathbf{S}$ as a quasivariety. On the other hand, if $\Gamma \models_{\mathbf{S}} \varphi$ for some finite $\Gamma \cup\{\varphi\} \subseteq \operatorname{Fm}_{\mathcal{L}}$ where $\{\rightarrow\} \subseteq \mathcal{L} \subseteq\{\rightarrow, \cdot, \neg\}$, then, since $\mathbb{V}\left(\mathrm{SA}_{\mathrm{m}}\right)=\mathbb{V}(\mathbf{S})$, the rule $\Gamma / \varphi$ is $\operatorname{RM}\lceil\mathcal{L}$-admissible. In particular:

Lemma 5.2. (A) is $\mathrm{RM}\lceil\mathcal{L}$-admissible for $\{\rightarrow\} \subseteq \mathcal{L} \subseteq\{\rightarrow, \cdot, \neg\}$.

Our initial goal will be to show that $\{(\mathrm{A})\}$ in fact constitutes a basis for the admissible rules of the $\{\rightarrow\}$ and $\{\rightarrow, \cdot\}$ fragments of RM. First, we note that, as shown in a more general setting by Avron in [5], the connective $\Rightarrow$ provides a deduction theorem with respect to $\mathbf{S}$.

Lemma 5.3 (see [5], Theorem III.2.2). For $\Gamma \cup\{\varphi\} \subseteq \operatorname{Fm}_{\{\rightarrow, \cdot, \neg\}}$ :

$$
\Gamma \cup\{\varphi\} \models_{\mathbf{S}} \psi \quad \text { iff } \quad \Gamma \models_{\mathbf{S}} \varphi \Rightarrow \psi .
$$

Proof. $(\Rightarrow)$ Suppose that $\Gamma \cup\{\varphi\} \models_{\mathbf{S}} \psi$ and that each formula in $\Gamma$ is evaluated as 0 or 1 in $\mathbf{S}$. If $\varphi$ is evaluated as 0 or 1 , then $\psi$ is evaluated as 0 or 1 , and therefore so also is $\varphi \Rightarrow \psi$. (The only tricky case when $\varphi$ is 1 and $\psi$ is 0 follows from the fact that $|\psi|$ is then 0 and so $\varphi \rightarrow|\psi|$ and $\varphi \rightarrow \psi$ are both -1 .) If $\varphi$ is evaluated as -1 , then $\varphi \Rightarrow \psi$ is clearly evaluated as 0 or 1 .

$(\Leftarrow)$ If $\Gamma \models_{\mathbf{S}} \varphi \Rightarrow \psi$, then $\Gamma \cup\{\varphi\} \models_{\mathbf{S}} \varphi \Rightarrow \psi$ and since also $\Gamma \cup\{\varphi\} \models_{\mathbf{S}} \varphi$ and $\{\varphi, \varphi \Rightarrow \psi\} \models_{\mathbf{S}} \psi$, it follows that $\Gamma \cup\{\varphi\} \models_{\mathbf{S}} \psi$.

Similarly to the connective $\supset$ defined in Section 3, which provides a deduction theorem for fragments of RM containing both $\rightarrow$ and $\vee$, the connective $\Rightarrow$ may be understood as incorporating a form of implication with weakening. Note, however, that these two implications do not coincide; e.g., evaluating $p$ as 2 and $q$ as -1 in $\mathbf{Z}$, we have that $p \Rightarrow q$ is 2 and that $p \supset q$ is -1 .

Next, adapting similar proofs by Avron in $[4,5]$, we obtain an axiomatization of the logic with equivalent quasivariety $\mathbb{Q}(\mathbf{S})$.

Lemma 5.4. $\mathrm{RM}_{\mathrm{m}}+(\mathrm{A})$ has equivalent quasivariety $\mathbb{Q}(\mathbf{S})$. 
Proof. It suffices to show that for any $\Gamma=\left\{\psi_{1}, \ldots, \psi_{n}\right\} \subseteq \operatorname{Fm}_{\{\rightarrow, \cdot,\urcorner\}}$ and $\varphi \in \operatorname{Fm}_{\{\rightarrow,,, \neg\}}$ :

$$
\Gamma \vdash_{\mathrm{RM}_{\mathrm{m}}+(\mathrm{A})} \varphi \quad \text { iff } \quad \Gamma \models_{\mathbf{S}} \varphi .
$$

$(\Rightarrow)$ If $\Gamma \vdash_{\mathrm{RM}_{\mathrm{m}}} \varphi$, then $\Gamma \models_{\mathbf{S}} \varphi$ since $\mathbf{S}$ is the reduct of a Sugihara algebra. Also $\{p, p \Rightarrow q\} \models_{\mathbf{S}} q$. Hence the logic defined by $\models_{\mathbf{S}}$ contains $\mathrm{RM}_{\mathrm{m}} \cup\{(\mathrm{A})\}$. So $\Gamma \vdash_{\mathrm{RM}_{\mathrm{m}}+(\mathrm{A})} \varphi$ implies $\Gamma \models_{\mathbf{S}} \varphi$.

$(\Leftarrow)$ Suppose that $\Gamma \models_{\mathbf{S}} \varphi$, and let $\vec{\psi} \Rightarrow \varphi$ denote the formula $\psi_{1} \Rightarrow\left(\psi_{2} \Rightarrow \ldots \supset\left(\psi_{n} \Rightarrow \varphi\right) \ldots\right)$. Then by Lemma $5.3, \models_{\mathrm{S}} \vec{\psi} \Rightarrow \varphi$. So by Theorem 5.1, $\vdash_{\mathrm{RM}_{\mathrm{m}}} \vec{\psi} \Rightarrow \varphi$ and hence $\vdash_{\mathrm{RM}_{\mathrm{m}}+\text { (A) }} \vec{\psi} \Rightarrow \varphi$. But also $\Gamma \vdash_{\mathrm{RM}_{\mathrm{m}}+\text { (A) }} \psi_{i}$ for $i=1 \ldots n$. Hence, using the rule (A) $n$ times, we obtain $\Gamma \vdash_{\mathrm{RM}_{\mathrm{m}}+\text { (A) }} \varphi$ as required.

Moreover, for the implication and implication-fusion fragments of RM, adding the rule (A) also secures structural completeness.

Lemma 5.5. $\operatorname{RM} \uparrow\{\rightarrow\}+(\mathrm{A})$ and $\operatorname{RM} \uparrow\{\rightarrow, \cdot\}+(\mathrm{A})$ are structurally complete.

Proof. By Lemma 2.3, it suffices to show that $h_{\mathbb{Q}(\mathbf{S} \mid\{\rightarrow, \cdot\})} \circ g$ embeds $\mathbf{S}\left\lceil\{\rightarrow, \cdot\}\right.$ into $\mathbf{F}_{\mathbb{Q}(\mathbf{S} \mid\{\rightarrow, \cdot\})}$ where $g:\{-1,0,1\} \rightarrow \mathrm{Fm}_{\{\rightarrow\}}$ is defined by:

$$
\begin{aligned}
g(1) & =(p \rightarrow q) \rightarrow(p \rightarrow q) \\
g(0) & =q \rightarrow q \\
g(-1) & =((p \rightarrow q) \rightarrow(p \rightarrow q)) \rightarrow(q \rightarrow q) .
\end{aligned}
$$

First we check (e.g., using Avron's sequent calculus [3]) that the image of $\mathbf{S}\left\lceil\{\rightarrow, \cdot\}\right.$ under $h_{\mathbb{Q}(\mathbf{S} \backslash\{\rightarrow, \cdot\})^{\circ}}$ $g$ behaves appropriately with respect to $\cdot$. For all formulas $\varphi, \psi \in \operatorname{Fm}_{\{\rightarrow, \cdot, \neg\}}$, we have $\models_{\mathbf{S}} \varphi \approx \varphi \cdot \varphi$ and $\models_{\mathbf{S}} \varphi \cdot \psi \approx \psi \cdot \varphi$. Hence we need only check that $\models_{\mathbf{S}} g(1) \cdot g(0) \approx g(1), \models_{\mathbf{S}} g(-1) \cdot g(0) \approx g(-1)$, and $=_{\mathbf{S}} g(1) \cdot g(-1) \approx g(-1)$, i.e.

$$
\begin{aligned}
& \models_{\mathbf{S}}((p \rightarrow q) \rightarrow(p \rightarrow q)) \cdot(q \rightarrow q) \approx(p \rightarrow q) \rightarrow(p \rightarrow q) \\
& { }_{\mathbf{S}}(((p \rightarrow q) \rightarrow(p \rightarrow q)) \rightarrow(q \rightarrow q)) \cdot(q \rightarrow q) \approx((p \rightarrow q) \rightarrow(p \rightarrow q)) \rightarrow(q \rightarrow q) \\
& { }_{\mathbf{S}}((p \rightarrow q) \rightarrow(p \rightarrow q)) \cdot(((p \rightarrow q) \rightarrow(p \rightarrow q)) \rightarrow(q \rightarrow q)) \approx((p \rightarrow q) \rightarrow(p \rightarrow q)) \rightarrow(q \rightarrow q) .
\end{aligned}
$$

Similarly, we may check (again using Avron's sequent calculus [3]) that the image of $\mathbf{S} \uparrow\{\rightarrow, \cdot\}$ under $h_{\mathbb{Q}(\mathbf{S} \backslash\{\rightarrow, \cdot\})} \circ g$ behaves appropriately with respect to $\rightarrow$. That is, we check that for each $a, b \in\{-1,0,1\}$ :

$$
\models_{\mathbf{S}} g(a) \rightarrow g(b) \approx g(a \rightarrow b) .
$$

Finally, we observe that $h_{\mathbb{Q}(\mathbf{S} \mid\{\rightarrow, \cdot\})} \circ g$ is 1-1: there are homomorphisms $h: \mathbf{F m}_{\{\rightarrow, \cdot\}} \rightarrow \mathbf{S}$ that distinguish the formulas in the image of $g$.

Since (A) is admissible for the $\{\rightarrow\}$ and $\{\rightarrow, \cdot\}$ fragments of RM (Lemma 5.2), we obtain immediately from Lemma 2.1:

Theorem 5.6. $\{(\mathrm{A})\}$ is a basis for the admissible rules of the $\{\rightarrow\}$ and $\{\rightarrow, \cdot\}$ fragments of $\mathrm{RM}$.

We turn our attention now to the multiplicative fragment $\mathrm{RM}_{\mathrm{m}}$. The following defined biimplication will be useful:

$$
\varphi \leftrightarrow \psi==_{\text {def }}(\varphi \rightarrow \psi) \cdot(\psi \rightarrow \varphi) .
$$

Observe that $\varphi \leftrightarrow \psi$ is -1 in $\mathbf{S}$ if $\varphi$ and $\psi$ take different values, 0 if they both take value 0 , and 1 if they both take the same value -1 or 1 . We write $\varphi_{1} \leftrightarrow \varphi_{2} \leftrightarrow \varphi_{3} \ldots \leftrightarrow \varphi_{n}$ for $\left(\ldots\left(\varphi_{1} \leftrightarrow \varphi_{2}\right) \leftrightarrow \varphi_{3} \ldots\right) \leftrightarrow \varphi_{n}$ and consider the following rules for $n \in \mathbb{N} \backslash\{0\}$ :

$$
\left(\mathrm{R}_{n}\right) \quad\left\{\neg\left(\left(p_{1} \rightarrow p_{1}\right) \leftrightarrow \ldots \leftrightarrow\left(p_{n} \rightarrow p_{n}\right)\right)\right\} / q .
$$

Note that in $\mathbf{S}$, for all $a_{1}, \ldots, a_{n} \in\{-1,0,1\}$ :

$$
\neg\left(\left(a_{1} \rightarrow a_{1}\right) \leftrightarrow \ldots \leftrightarrow\left(a_{n} \rightarrow a_{n}\right)\right)= \begin{cases}0 \text { or } 1 & \text { if } 0 \in\left\{a_{1}, \ldots, a_{n}\right\} \\ -1 & \text { otherwise. }\end{cases}
$$

For convenience, let us call this property $(*)$. 
Lemma 5.7. $\left(\mathrm{R}_{n}\right)$ is $\mathrm{RM}_{\mathrm{m}}$-admissible for each $n \in \mathbb{N} \backslash\{0\}$.

Proof. No substitution makes the premise of $\left(\mathrm{R}_{n}\right)(n \in \mathbb{N} \backslash\{0\})$ into a theorem of classical logic. Hence the same also holds for $\mathrm{RM}_{\mathrm{m}}$, and each $\left(\mathrm{R}_{n}\right)(n \in \mathbb{N} \backslash\{0\})$ is $\mathrm{RM}_{\mathrm{m}}$-admissible.

Consider now the subalgebra $\mathbf{2}=\langle\{-1,1\}, \rightarrow, \cdot, \neg\rangle$ of $\mathbf{S}$, term-equivalent to the two element Boolean algebra. Since $\mathbf{S}$ is a homomorphic image of $\mathbf{2} \times \mathbf{S}$, we have $\mathbb{V}(\mathbf{2} \times \mathbf{S})=\mathbb{V}(\mathbf{S})$. In what follows, we will show that a particular axiomatization of $\mathbb{Q}(\mathbf{2} \times \mathbf{S})$ is structurally complete and hence provides a basis for the admissible rules of $\mathrm{RM}_{\mathrm{m}}$.

Lemma 5.8. $\mathrm{RM}_{\mathrm{m}}+(\mathrm{A})+\left\{\left(\mathrm{R}_{n}\right) \mid n \in \mathbb{N} \backslash\{0\}\right\}$ has equivalent quasivariety $\mathbb{Q}(\mathbf{2} \times \mathbf{S})$.

Proof. For convenience, let

$$
\mathrm{L}=\mathrm{RM}_{\mathrm{m}}+(\mathrm{A})+\left\{\left(\mathrm{R}_{n}\right) \mid n \in \mathbb{N} \backslash\{0\}\right\} .
$$

Then it suffices to show that for any finite $\Gamma \cup\{\varphi\} \subseteq \operatorname{Fm}_{\{\rightarrow, \cdot, \neg\}}$ :

$$
\Gamma \vdash_{\mathrm{L}} \varphi \quad \text { iff } \quad \Gamma \models_{\mathbf{2} \times \mathbf{S}} \varphi \text {. }
$$

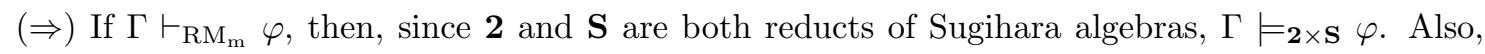
$\{p, p \Rightarrow q\} \models_{\mathbf{2} \times \mathbf{S}} q$ as $\{p, p \Rightarrow q\} \models_{\mathbf{2}} q$ and $\{p, p \Rightarrow q\} \models_{\mathbf{S}} q$. Finally, $\left\{\neg\left(\left(p_{1} \rightarrow p_{1}\right) \leftrightarrow \ldots \leftrightarrow\right.\right.$ $\left.\left.\left(p_{n} \rightarrow p_{n}\right)\right)\right\} \models \mathbf{2} \times \mathbf{S} q$ for each $n \in \mathbb{N} \backslash\{0\}$ since $\neg\left(\left(p_{1} \rightarrow p_{1}\right) \leftrightarrow \ldots \leftrightarrow\left(p_{n} \rightarrow p_{n}\right)\right)$ is not satisfiable in 2. That is, the logic defined by $\models_{\mathbf{2} \times \mathbf{S}}$ contains $\operatorname{RM}_{\mathrm{m}} \cup\{(\mathrm{A})\} \cup\left\{\left(\mathrm{R}_{n}\right) \mid n \in \mathbb{N} \backslash\{0\}\right\}$. So $\Gamma \vdash_{\mathrm{L}} \varphi$ implies $\Gamma \models \mathbf{2} \times \mathbf{S} \varphi$.

$(\Leftarrow)$ Suppose that $\Gamma \models_{\mathbf{2} \times \mathbf{S}} \varphi$, where $\Gamma$ contains the variables $p_{1}, \ldots, p_{n}$. If $\Gamma \models \mathbf{S} \varphi$, then $\Gamma \vdash_{L} \varphi$ since $\mathbb{Q}(\mathbf{S})$ is an equivalent quasivariety for $\mathrm{RM}_{\mathrm{m}}+(\mathrm{A})$ (Lemma 5.4). Suppose then that $\Gamma \not \forall_{\mathbf{S}} \varphi$. I.e., there is a homomorphism $e: \mathbf{F m}_{\{\rightarrow, \cdot, \neg\}} \rightarrow \mathbf{S}$ such that $e(\psi) \geq 0$ for all $\psi \in \Gamma$ and $e(\varphi)=-1$. By assumption, $\Gamma \models_{\mathbf{2} \times \mathbf{S}} \varphi$. Hence $\Gamma$ is not satisfiable in $\mathbf{2}$. Otherwise, for some $k: \mathbf{F m}_{\{\rightarrow, \cdot, \neg\}} \rightarrow \mathbf{2}$, we have $k(\psi)=1$ for all $\psi \in \Gamma$ and we could define $j: \mathbf{F m}_{\{\rightarrow, \cdot, \neg\}} \rightarrow \mathbf{2} \times \mathbf{S}$ by $j(p)=(k(p), e(p))$ such that $j(\psi) \geq(1,0)$ for all $\psi \in \Gamma$ and $j(\varphi) \nsupseteq(1,0)$.

Now consider any homomorphism $h: \mathbf{F m}_{\{\rightarrow, \cdot, \neg\}} \rightarrow \mathbf{S}$ satisfying $\Gamma$ : i.e., such that $h(\psi) \geq 0$ for all $\psi \in \Gamma$. Since $\Gamma$ is not satisfiable in the subalgebra $\mathbf{2}$ of $\mathbf{S}$, we must have $h\left(p_{i}\right)=0$ for some $i \in\{1, \ldots, n\}$. But then, making use of property $(*)$ above:

$$
\Gamma \models_{\mathbf{S}} \neg\left(\left(p_{1} \rightarrow p_{1}\right) \leftrightarrow \ldots \leftrightarrow\left(p_{n} \rightarrow p_{n}\right)\right) .
$$

Hence $\Gamma \vdash_{\mathrm{RM}_{\mathrm{m}}+(\mathrm{A})} \neg\left(\left(p_{1} \rightarrow p_{1}\right) \leftrightarrow \ldots \leftrightarrow\left(p_{n} \rightarrow p_{n}\right)\right)$ by Lemma 5.4 , and clearly $\Gamma \vdash_{\mathrm{L}} \neg\left(\left(p_{1} \rightarrow\right.\right.$ $\left.\left.p_{1}\right) \leftrightarrow \ldots \leftrightarrow\left(p_{n} \rightarrow p_{n}\right)\right)$. Therefore, finally, using $\left(\mathrm{R}_{n}\right), \Gamma \vdash_{\mathrm{L}} \varphi$, as required.

Lemma 5.9. $\mathrm{RM}_{\mathrm{m}}+(\mathrm{A})+\left\{\left(\mathrm{R}_{n}\right) \mid n \in \mathbb{N} \backslash\{0\}\right\}$ is structurally complete.

Proof. By Lemmas 5.8 and 2.3, it suffices to show that $h_{\mathbb{Q}(\mathbf{2} \times \mathbf{S})} \circ g$ embeds $\mathbf{2} \times \mathbf{S}$ into $\mathbf{F}_{\mathbb{Q}(\mathbf{2} \times \mathbf{S})}$ where $g:\{-1,1\} \times\{-1,0,1\} \rightarrow \mathrm{Fm}_{\{\rightarrow, \neg\}}$ is defined by: ${ }^{1}$

$$
\begin{aligned}
g((-1,-1)) & =\neg((p \rightarrow q) \rightarrow(p \rightarrow q)) \\
g((-1,0)) & =\neg(p \rightarrow p) \\
g((-1,1)) & =\neg((q \rightarrow q) \rightarrow(p \rightarrow p)) \\
g((1,-1)) & =(q \rightarrow q) \rightarrow(p \rightarrow p) \\
g((1,0)) & =p \rightarrow p \\
g((1,1)) & =(p \rightarrow q) \rightarrow(p \rightarrow q) .
\end{aligned}
$$

Recall that $\mathbb{V}(\mathbf{2} \times \mathbf{S})=\mathbb{V}(\mathbf{S})$, so in particular $\mathbf{F}_{\mathbb{Q}(\mathbf{2} \times \mathbf{S})}=\mathbf{F}_{\mathrm{SA}_{\mathrm{m}}}$.

It is almost immediate to see that $h_{\mathbb{Q}(\mathbf{2} \times \mathbf{S})} \circ g$ respects the negation of $\mathbf{2} \times \mathbf{S}$. To show that $h_{\mathbb{Q}(\mathbf{2} \times \mathbf{S})} \circ g$ preserves $\cdot$, we can use the commutativity and idempotence of $\cdot$ in Sugihara

\footnotetext{
${ }^{1}$ This embedding was in fact discovered automatically using an algorithm described in [23].
} 
algebras; hence, it suffices to check (using, e.g., Avron's sequent calculus [3]) that for all $x \in$ $\{-1,1\} \times\{-1,0,1\}:$

$$
\begin{array}{rr}
\models_{\mathbf{S}} \quad g((-1,-1)) \cdot g(x) & \approx g((-1,-1)) \\
\models & =g((1,0)) \cdot g(x)
\end{array}
$$

and also

$$
\begin{aligned}
& \models_{\mathbf{S}} \quad g((-1,0)) \cdot g((-1,1)) \approx g((-1,1)) \\
& \models_{\mathrm{S}} \quad g((-1,0)) \cdot g((1,-1)) \approx g((-1,-1)) \\
& \models_{\mathbf{S}} \quad g((-1,0)) \cdot g((1,1)) \approx g((-1,1)) \\
& \models_{\mathbf{S}} \quad g((-1,1)) \cdot g((1,-1)) \approx g((-1,-1)) \\
& \models_{\mathbf{S}} \quad g((-1,1)) \cdot g((1,1)) \approx g((-1,1)) \\
& \models_{\mathbf{S}} \quad g((1,-1)) \cdot g((1,1)) \approx g((1,-1)) .
\end{aligned}
$$

Since $x \rightarrow y \approx \neg(x \cdot \neg y)$ holds in all Sugihara algebras, $h_{\mathbb{Q}(\mathbf{2} \times \mathbf{S})} \circ g$ also preserves $\rightarrow$. Finally, $h_{\mathbb{Q}(\mathbf{2} \times \mathbf{S})} \circ g$ is 1-1: we can find homomorphisms $h: \mathbf{F m}_{\{\rightarrow, \cdot, \neg\}} \rightarrow \mathbf{S}$ that distinguish the formulas in the image of $g$.

Since $(\mathrm{A})$ and $\left(\mathrm{R}_{n}\right)(n \in \mathbb{N} \backslash\{0\})$ are $\mathrm{RM}_{\mathrm{m}}$-admissible (Lemmas 5.2 and 5.7), we obtain immediately from Lemma 2.1:

Theorem 5.10. The rules (A) and $\left\{\left(\mathrm{R}_{n}\right) \mid n \in \mathbb{N} \backslash\{0\}\right\}$ form a basis for the admissible rules of the multiplicative fragment of RM.

The natural question to ask at this point is whether a finite set of rules would suffice. This is answered negatively as follows:

Theorem 5.11. The admissible rules of the multiplicative fragment of RM have no finite basis.

Proof. By compactness, any finite set of rules derivable in $\mathrm{RM}_{\mathrm{m}}+(\mathrm{A})+\left\{\left(\mathrm{R}_{n}\right) \mid n \in \mathbb{N} \backslash\{0\}\right\}$ is derivable in $\mathrm{RM}_{\mathrm{m}}+(\mathrm{A})+\left\{\left(\mathrm{R}_{n}\right) \mid 1 \leq n \leq k\right\}$ for some $k \in \mathbb{N} \backslash\{0\}$. Hence it suffices to show that for each $k \in \mathbb{N} \backslash\{0\}$, the rules (A) and $\left\{\left(\mathrm{R}_{n}\right) \mid 1 \leq n<k\right\}$ do not form a basis for the admissible rules of $\mathrm{RM}_{\mathrm{m}}$. Consider the following subalgebra of $\mathbf{S}^{\mathbf{k}}$ :

$$
\mathbf{A}=\left\{\left(\beta_{1}, \ldots, \beta_{k}\right) \in\{-1,0,1\}^{k} \mid \text { at most one of the } \beta_{i} \text { 's is } 0\right\} .
$$

We will prove that:

$$
(\star) \quad\left\{\neg\left(\left(p_{1} \rightarrow p_{1}\right) \leftrightarrow \ldots \leftrightarrow\left(p_{n} \rightarrow p_{n}\right)\right)\right\} \models_{\mathbf{A}} q \quad \text { iff } \quad n<k .
$$

It follows from $(\star)$ that $\mathbf{A}$ belongs to the equivalent quasivariety for $\mathrm{RM}_{\mathrm{m}}+(\mathrm{A})+\left\{\left(\mathrm{R}_{n}\right) \mid 1 \leq n<\right.$ $k\}$, but not to $\mathbb{Q}(\mathbf{2} \times \mathbf{S})$, the equivalent quasivariety for $\mathrm{RM}_{\mathrm{m}}+(\mathrm{A})+\left\{\left(\mathrm{R}_{n}\right) \mid n \in \mathbb{N} \backslash\{0\}\right\}$. But then the rules (A) and $\left\{\left(\mathrm{R}_{n}\right) \mid 1 \leq n<k\right\}$ cannot form a basis for the admissible rules of $\mathrm{RM}_{\mathrm{m}}$.

To prove $(\star)$, note first that for each $n \in \mathbb{N} \backslash\{0\}$ :

$$
\left\{\neg\left(\left(p_{1} \rightarrow p_{1}\right) \leftrightarrow \ldots \leftrightarrow\left(p_{n} \rightarrow p_{n}\right)\right)\right\} \not \forall_{\mathbf{A}} q
$$

iff for some homomorphism $h: \operatorname{Fm}_{\{\rightarrow, \cdot, \neg\}} \rightarrow \mathbf{A}$,

$$
h\left(\neg\left(\left(p_{1} \rightarrow p_{1}\right) \leftrightarrow \ldots \leftrightarrow\left(p_{n} \rightarrow p_{n}\right)\right)\right) \geq(0, \ldots, 0),
$$

i.e., for some $a_{1}, \ldots, a_{n} \in A$ and $\left(\beta_{1}, \ldots, \beta_{k}\right)=\neg\left(\left(a_{1} \rightarrow a_{1}\right) \leftrightarrow \ldots \leftrightarrow\left(a_{n} \rightarrow a_{n}\right)\right)$, we have $\beta_{i} \geq 0$ for $i=1 \ldots k$. Equivalently, by property $(*)$, there should exist $a_{1}, \ldots, a_{n} \in A$ such that for each $i=1 \ldots k$, we have $a_{j}=\left(\alpha_{1}, \ldots, \alpha_{i-1}, 0, \alpha_{i+1}, \ldots, \alpha_{k}\right)$ for some $j \in\{1, \ldots, n\}$. But $A$ consists of all those members of $S^{k}$ with at most one 0 . So the latter holds iff $n \geq k$, and we obtain $(\star)$.

Moreover, since the admissible rules of $\mathrm{RM}_{\mathrm{m}}+(\mathrm{A})$ coincide with the admissible rules of $\mathrm{RM}_{\mathrm{m}}$, we also obtain:

Corollary 5.12. The admissible rules of the finitely axiomatizable three-valued logic $\mathrm{RM}_{\mathrm{m}}+(\mathrm{A})$ have no finite basis. 
Table 1: Admissibility in proper fragments of RM.

\begin{tabular}{lll}
\hline \hline Fragment & Admissible Rules & Reference \\
\hline$\{\rightarrow, \wedge\}$ & Hereditarily structurally complete & Theorem 4.1 \\
$\{\rightarrow, \cdot, \vee\}$ & Hereditarily structurally complete & Theorem 4.1 \\
$\{\rightarrow, \vee\}$ & Hereditarily structurally complete & Theorem 4.1 \\
$\{\rightarrow, \cdot\}$ & Basis: $\{(\mathrm{A})\}$ & Theorem 5.6 \\
$\{\rightarrow\}$ & Basis: $\{(\mathrm{A})\}$ & Theorem 5.6 \\
$\{\rightarrow, \neg\}$ & Basis: $\{(\mathrm{A})\} \cup\left\{\left(\mathrm{R}_{n}\right) \mid n \in \mathbb{N} \backslash\{0\}\right\}$ & Theorem 5.10 \\
\hline \hline
\end{tabular}

This contrasts with the fact, established by Rautenberg in [28], that every two-valued logic is both finitely axiomatizable and hereditarily structurally complete. Examples of tabular intermediate and modal logics with more than three values whose admissible rules are not finitely axiomatizable are given by Rybakov in [31], but to the best of our knowledge, $\mathrm{RM}_{\mathrm{m}}+(\mathrm{A})$ is the first example of a three-valued logic having this property.

Alternative bases for the admissible rules of the multiplicative and implication-fusion fragments are provided by swapping (A) for $\{p \cdot q\} / q$, since as shown by Avron in [4], $\mathbb{Q}(\mathbf{S})$ is an equivalent quasivariety for $\mathrm{RM}_{\mathrm{m}}$ extended with this rule. Finally, the situation for proper fragments of Rmingle is summarized in Table 1, recalling that the full logic is not structurally complete but that no basis for its admissible rules has yet been found.

\section{References}

[1] A. R. Anderson and N. D. Belnap, Entailment, Vol. 1, Princeton Univ. Press, 1975.

[2] A. Avron, On an implication connective of RM, Notre Dame J. Form. Log. 27 (1986), 201-209.

[3] _ A constructive analysis of RM, J. Symbolic Logic 52 (1987), no. 4, 939-951.

[4] _ Multiplicative conjunction as an extensional conjunction, Log. J. IGPL 5 (1997), 181-208.

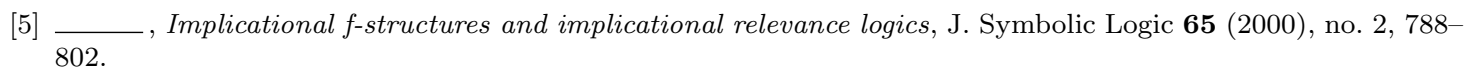

[6] S. Babenyshev and V. Rybakov, Linear temporal logic LTL: Basis for admissible rules, J. Log. Comput. 21 (2011), no. 2, 157-177.

[7] __ Unification in linear temporal logic LTL, Ann. Pure Appl. Logic 162 (2011), no. 12, 991-1000.

[8] W. J. Blok and D. Pigozzi, Algebraizable logics, Mem. Amer. Math. Soc., vol. 77, Amer. Math. Soc., 1989.

[9] W. J. Blok and J. G. Raftery, Fragments of R-mingle, Studia Logica 78 (2004), no. 1-2, 59-106.

[10] L. M. Cabrer and G. Metcalfe, Admissibility via natural dualities. Submitted.

[11] P. Cintula and G. Metcalfe, Structural completeness in fuzzy logics, Notre Dame J. Form. Log. 50 (2009), no. 2, 153-183.

[12] Admissible rules in the implication-negation fragment of intuitionistic logic, Ann. Pure Appl. Logic 162 (2010), no. 10, 162-171.

[13] J. M. Dunn, Algebraic completeness for R-mingle and its extensions, J. Symbolic Logic 35 (1970), 1-13.

[14] S. Ghilardi, Unification in intuitionistic logic, J. Symbolic Logic 64 (1999), no. 2, 859-880.

[15] _ _ Best solving modal equations, Ann. Pure Appl. Logic 102 (2000), no. 3, $184-198$.

[16] R. Iemhoff, On the admissible rules of intuitionistic propositional logic, J. Symbolic Logic 66 (2001), no. 1, $281-294$.

[17] _ Intermediate logics and Visser's rules, Notre Dame J. Form. Log. 46 (2005), no. 1, 65-81.

[18] R. Iemhoff and G. Metcalfe, Proof theory for admissible rules, Ann. Pure Appl. Logic 159 (2009), no. 1-2, 171-186.

[19] E. Jeřábek, Admissible rules of modal logics, J. Logic Comput. 15 (2005), 411-431.

[20] __ Admissible rules of Eukasiewicz logic, J. Logic Comput. 20 (2010), no. 2, 425-447.

[21] _ Bases of admissible rules of Eukasiewicz logic, J. Logic Comput. 20 (2010), no. 6, 1149-1163. 
[22] G. Metcalfe and C. Röthlisberger, Admissibility in De Morgan algebras, Soft Comput. 16 (2012), no. 11, 18751882 .

[23] _ Admissibility in finitely generated quasivarieties, Log. Methods Comput. Sci. 9 (2013), no. 2, 1-19.

[24] J. S. Olson and J. G. Raftery, Positive Sugihara monoids, Algebra Universalis 57 (2007), 75-99.

[25] J. S. Olson, J. G. Raftery, and C. J. Van Alten, Structural completeness in substructural logics, Log. J. IGPL 16 (2008), no. 5, 453-495.

[26] T. Prucnal, On the structural completeness of some pure implicational propositional calculi, Studia Logica 32 (1973), no. 1, 45-50.

[27] J. G. Raftery, Representable idempotent commutative residuated lattices, Trans. Amer. Math. Soc. 359 (2007), $4405-4427$.

[28] W. Rautenberg, 2-element matrices, Studia Logica 40 (1981), no. 4, 315-353 (1982).

[29] P. Rozière, Regles admissibles en calcul propositionnel intuitionniste, Ph.D. Thesis, 1992.

[30] V. Rybakov, A criterion for admissibility of rules in the modal system S4 and the intuitionistic logic, Algebra Logic 23 (1984), 369-384.

[31] _ Admissibility of logical inference rules, Studies in Logic and the Foundations of Mathematics, vol. 136, Elsevier, Amsterdam, 1997.

[32] J. K. Slaney and R. K. Meyer, A structurally complete fragment of relevant logic, Notre Dame J. Form. Log. 33 (1992), 561-566.

[33] B. Sobociński, Axiomatization of a partial system of three-valued calculus of propositions, The Journal of Computing Systems 1 (1952), 23-55. 